\title{
Diseño de un clasificador para pacientes en proceso de extubación
}

\section{Classifier design for patients on weaning process}

\author{
Hernando González-Acevedo \\ M. Sc. Ingeniería \\ Universidad Autónoma de Bucaramanga \\ Bucaramanga, Colombia \\ hgonzalez7@unab.edu.co
}

\author{
Carlos Julio Arizmendi-Pereira \\ Ph. D. Inteligencia Artificial \\ Universidad Autónoma de Bucaramanga \\ Bucaramanga, Colombia \\ carizmendi@unab.edu.co
}

\author{
Beatriz Giraldo-Giraldo \\ Ph. D. Ingeniería Biomédica \\ Universidad Politécnica de Catalunya \\ Barcelona, España \\ beatriz.giraldo@upc.edu
}

\begin{abstract}
Resumen- La ventilación mecánica (VM) es una estrategia terapéutica que consiste en asistir o reemplazar mecánicamente la ventilación pulmonar espontánea. Con el objetivo de desarrollar un software de apoyo para los médicos, se realizó un estudio de las señales respiratorias, utilizando la transformada de wavelet discreta, para determinar los descriptores que indiquen si el paciente puede ser desconectado del ventilador mecánico. Para reducir la dimensionalidad del sistema se realizó un análisis de componentes principales (PCA), determinando tres variables óptimas, las cuales son las entradas a los clasificadores que se analizaron en el artículo: K-Nearest Neighbor y lógica difusa.
\end{abstract}

Palabras clave- Análisis de componentes principales, clasificador K-Nearest Neighbor, clasificador lógica difusa, transformada wavelet, ventilación mecánica.

Abstract- The mechanical ventilation (MV) is a therapeutic strategy to mechanically assist or replace spontaneous breathing. With the objective of developing a software support for doctors was performed a study of respiratory signals using the discrete wavelet transform to determine the descriptors to indicate whether the patient can be disconnected from the mechanical ventilator. To reduce the dimensionality of the system was performed a principal component analysis (PCA), establishing three variables optimal, which are the inputs to the classifiers that were analyzed in the article: K-Nearest Neighbor and fuzzy logic.

Keywords- Fuzzy logic classifier, K-Nearest neighbor classifier, mechanical ventilation, principal component analysis, wavelet transform.

\section{INTRODUCCIÓN}

Uno de los problemas más desafiantes en las unidades de cuidados intensivos es el proce- so de desconexión del paciente de la ventilación mecánica. Una prueba fallida es molesta para el paciente, y puede ocasionar distrés cardiopulmonar. En el proceso de interrupción de ventilación mecánica, hasta el $25 \%$ de pacientes sufren un distrés lo bastante severo como para requerir su reintubación. Por ello la necesidad de un pronóstico lo más exacto posible del momento óptimo de extubación, se extiende a todo el proceso de weaning o destete del paciente [1], [2], [3].

La variabilidad del patrón respiratorio es no randómica y ha sido objeto de estudio por diferentes investigadores [4], [5], [6], [7], [8], aplicando métodos lineales y modelos paramétricos. En [9] se proponen tres modelos diferentes: un modelo autorregresivo (AR), un modelo autorregresivo de media móvil (ARMA) y un modelo autorregresivo con entrada exógena (ARX); obteniendo una exactitud del $86 \%$ con el primer modelo. En [10] realizaron una caracterización de la dinámica del flujo respiratorio utilizando un modelo ARMA, logrando una exactitud del $75.3 \%$.

Con el objetivo de encontrar posibles diferencias entre pacientes que tras la prueba de extubación puedan mantener la respiración de forma espontánea, pacientes que fracasan en este intento, y pacientes que aun habiendo superado el proceso de destete o extubación deben ser reintubados antes de 48 horas. Se han trabajado las siguientes técnicas de clasificación utilizando las señales de flujo respiratorio: máquinas de soporte vectorial [11], [12] y redes neuronales [13], [14]. 
Otros autores han centrado sus investigaciones en determinar una relación entre el patrón respiratorio y la frecuencia cardiaca, en pacientes durante el proceso de destete, utilizando técnicas como dinámica simbólica [15], análisis multiparamétrico [16] y análisis de cuantificación recurrente [17].

\section{PROCESAMIENTO DE LAS SEÑALES RESPIRATORIAS MEDIANTE LA TRANSFORMADA WAVELET DISCRETA}

La base de datos WEAN DB es el resultado del estudio de las señales de flujo respiratorio de 133 pacientes asistidos mediante ventilación mecánica y en proceso de extubación. Los pacientes fueron registrados en los departamentos de Cuidados Intensivos del Hospital de la Santa Creu i Sant Pau de Barcelona, y del Hospital de Getafe, de acuerdo con los protocolos aprobados por los comités éticos. La señal de flujo respiratorio se registró a partir de un neumotacógrafo (monitor Datex-Ohmeda) conectado a un tubo endotraqueal, a una frecuencia de muestreo de $250 \mathrm{~Hz}$ (tarjeta de adquisición National Instruments DAQCard 6024E).

Los pacientes registrados fueron sometidos a la prueba de tubo en $\mathrm{T}$, con respiración espontánea durante 30 minutos, como protocolo para la extubación. Los pacientes que mantuvieron la respiración espontánea con normalidad fueron extubados, en caso contrario reconectados. De acuerdo con el criterio clínico, los pacientes fueron clasificados en dos grupos: grupo éxito (Clase C0), 94 pacientes (61 hombres y 33 mujeres) con éxito en el destete, y grupo fracaso (Clase C1), 39 pacientes (24 hombres y 15 mujeres) que no superaron la prueba y fueron conectados de nuevo al ventilador.

Para cada señal de flujo respiratorio correspondiente a un paciente se evaluaron los siguientes parámetros: duración del ciclo respiratorio (TTot=TI+TE), tiempo de inspiración (TI), tiempo de expiración (TE), el volumen circulante o volumen tidal (VT), la fracción inspiratoria (TI/TTot), el flujo inspirado medio (VT/TI) y la relación frecuencia-volumen tidal (f/VT). La Fig. 1 presenta los parámetros extraídos de las señales de flujo y volumen respiratorio para el estudio.

\subsection{La transformada Wavelet}

La transformada Wavelet de una función $\mathrm{f}(\mathrm{t})$ es la descomposición de la señal en un conjunto de funciones $\psi_{s, \tau}(t)$, que forman una base y son llamadas las wavelets, como se define en (1). Las wavelets son generadas a partir de la translación y cambio de escala de una misma función wavelet , llamada la Wavelet madre, dada por (2), donde $s$ es el factor de escalamiento y $\tau$ es el factor de desplazamiento en el tiempo.

$$
\begin{aligned}
W_{f}(s, \tau) & =\int f(t) \psi_{s, \tau}^{*}(t) d t \\
\psi_{s, \tau}(t) & =\frac{1}{\sqrt{s}} \psi\left(\frac{t-\tau}{s}\right)
\end{aligned}
$$

Para que la teoría de Wavelet sea útil, es necesario que se disponga de algoritmos rápidos para su uso en computadoras. Un método eficiente fue desarrollado en 1988 por Mallat para evaluar la transformada de Wavelet discreta; el método se fundamenta en descomponer la señal utilizando filtros con diferentes frecuencias de corte. En esta clase de análisis se habla de coeficientes de aproximación y coeficientes de detalle. Las aproximaciones son las componentes de baja frecuencia de la señal y los detalles son los componentes de alta frecuencia.

Para realizar el procesamiento de las señales que conforman la base de datos, en primer lugar se determina la función wavelet que mejor caracteriza cada variable, para lo cual se evaluaron 34 funciones madre utilizando la toolbox de Wavelet de Matlab. Para cada función se descompone la señal en tres niveles y se reconstruye nuevamente, calculando el error medio cuadrático (MSE) entre la señal original y la señal reconstruida. El procedimiento se aplicó a los 133 pacientes que conforman las clases $\mathrm{CO}$ y C1, para cada una de las 7 series, analizando un total de 931 señales. En la Tabla I se observan los resultados generales, indicando para cada variable la función wavelet que presenta el menor error medio cuadrático. De los resultados se observa que la familia Biorthogonal describe de forma adecuada las señales asociadas con el flujo respiratorio y al realizar un análisis modal la wavelet madre que mayor número de veces se repite es la bior3.5. 
Fig. 1. PARÁMETROS EXTRAIDOS DE LAS SEÑALES DE FLUJO Y VOLUMEN RESPIRATORIO. (A) TIEMPO DE INSPIRACIÓN (TI), TIEMPO DE EXPIRACIÓN (TE) Y DURACIÓN DEL CICLO RESPIRATORIO (TTOT). (B) VOLUMEN TIDAL (VT)
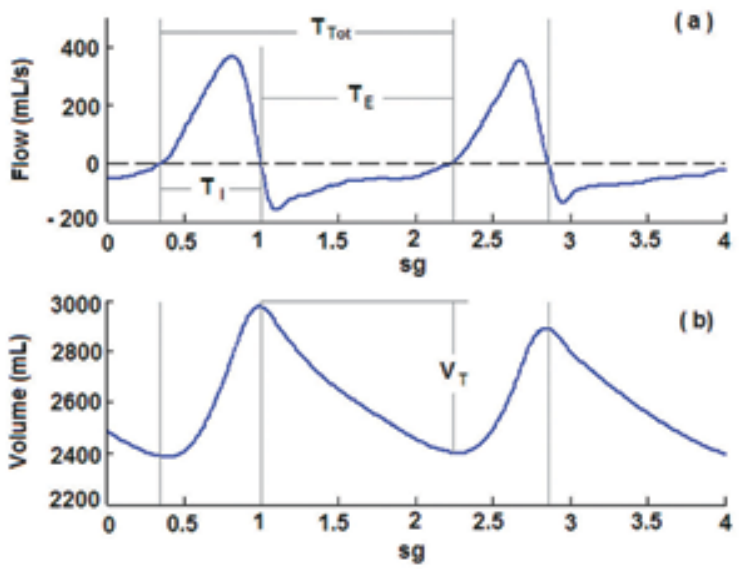

Fuente: autores.

TABLA I

PROMEDIO DEL MSE PARA LA CLASE CO Y C1

\begin{tabular}{|l|c|c|c|c|}
\cline { 2 - 5 } \multicolumn{1}{c|}{} & \multicolumn{2}{c|}{ Clase CO } & \multicolumn{2}{c|}{ Clase C1 } \\
\hline \multicolumn{1}{c|}{ Señal } & Wavelet Madre & Media MSE & Wavelet Madre & Media MSE \\
\hline SERIE f/VT & bior3.5 & 0.0095 & bior3.5 & 0.0068 \\
\hline SERIE TE & bior3.3 & 0.1302 & bior3.3 & 0.1905 \\
\hline SERIE TI & bior1.5 & 0.1119 & bior3.5 & 0.1016 \\
\hline SERIE TTot & bior3.3 & 0.0369 & bior3.5 & 0.0375 \\
\hline SERIE TI/TTot & bior3.5 & 0.2103 & bior3.7 & 0.2244 \\
\hline SERIE VT/TI & bior3.7 & 60.313 & bior3.5 & 61.1029 \\
\hline SERIE VT & bior3.7 & 47.8354 & bior3.5 & 69.6587 \\
\hline
\end{tabular}

Una vez seleccionada la función wavelet madre, se descompone cada señal en tres niveles y se calcula para los coeficientes de aproximación y detalle cinco parámetros estadísticos: la media, la desviación estándar, la varianza, el rango intercuartil y la oblicuidad. Se aplica el test de MannWhitney con el fin de definir cuál variable logra una mayor diferenciación entre clases. Este test es una prueba no paramétrica que permite verificar si las medianas de dos muestras son independientes. Cuando el $\mathrm{p}$ - value (valor que entrega la prueba) es menor a 0.05 se dice que existe una separación entre las medianas de cada clase. En la Fig. 2 se observa en un gráfico de barras los resultados al aplicar el test para cada uno de los parámetros estadísticos evaluados a los coeficientes de aproximación A3 y los coeficientes de detalle D1, D2 y D3. Las variables se encuentran codifi- cadas de la siguiente forma: 1 - Relación frecuencia-volumen, 2 - Tiempo de expiración, 3 - Tiempo de inspiración, 4 - Ciclo respiratorio, 5 - Fracción inspiratoria, 6 - Flujo inspirado medio, 7 - Volumen tidal. De las 3724 nuevas señales generadas, solo dieciocho presentaron un p-value menor a 0.05 . En los coeficientes de aproximación A3 cumplen con la condición la media de las señales f/VT, TE, TI y TI/TTot; la desviación estándar de la señal TI/TTot, la varianza de la señal TI/TTot, el rango intercuartil de la señal TI/TTot, la oblicuidad de la señal TE y la oblicuidad TI/TTot. De los coeficientes de detalle D2, pasan la prueba la desviación estándar de las señales TE y TI/TTot, la varianza de las señales TE y TI/TTot, y el rango intercuartil de las señales TE y TI/TTot. De los coeficientes de detalle $\mathrm{D} 3$, el rango intercuartil de las señales TE y TTot, y la oblicuidad de la señal TTot. 
Fig. 2. RESULTADOS TEST DE MANN-WHITNEY PARA COEFICIENTES DE APROXIMACIÓN Y DETALLE
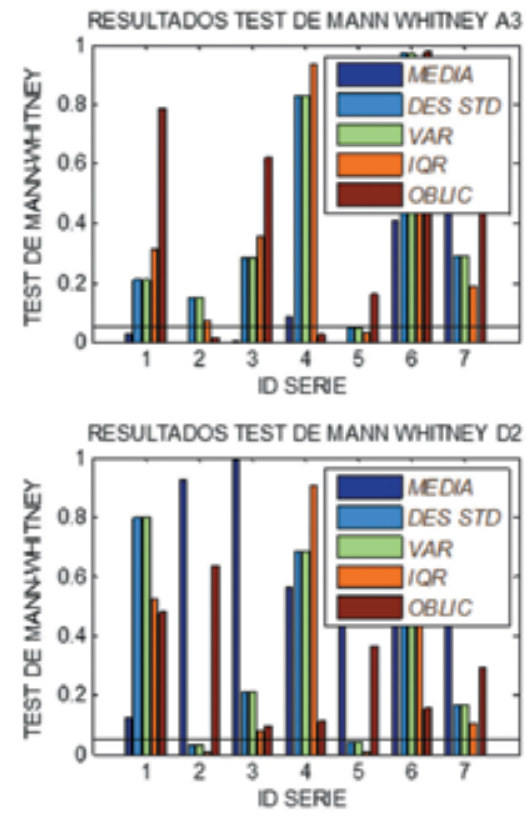

Fuente: autores.

\subsection{Reducción de dimensionalidad}

La reducción de dimensiones es frecuentemente usada como una etapa de pre-proceso en el entrenamiento de sistemas, y consiste en escoger un subconjunto de variables, de tal manera, que el espacio de características quede óptimamente reducido de acuerdo con un criterio de evaluación, cuyo fin es distinguir el subconjunto que representa mejor el espacio inicial de entrenamiento. El Análisis de Componentes Principales (PCA) es una técnica estadística para la reducción de la dimensión, desarrollada por Pearson a finales del siglo XIX y posteriormente estudiada por Hotelling en los años treinta del siglo XX. Para estudiar las relaciones que se presentan entre $\mathrm{p}$ variables correlacionadas (que miden información común) se puede transformar el conjunto original de variables en otro conjunto de nuevas variables incorreladas entre sí (que no tenga repetición o redundancia en la información), llamado conjunto de componentes principales. Las nuevas variables son combinaciones lineales de las anteriores y se van construyendo según el orden de importancia en cuanto a la variabilidad total que recogen de la muestra. Dada las 18 variables que cumplieron con el test de Mann-Whitney, se realizó una reducción de dimensionalidad, buscan-
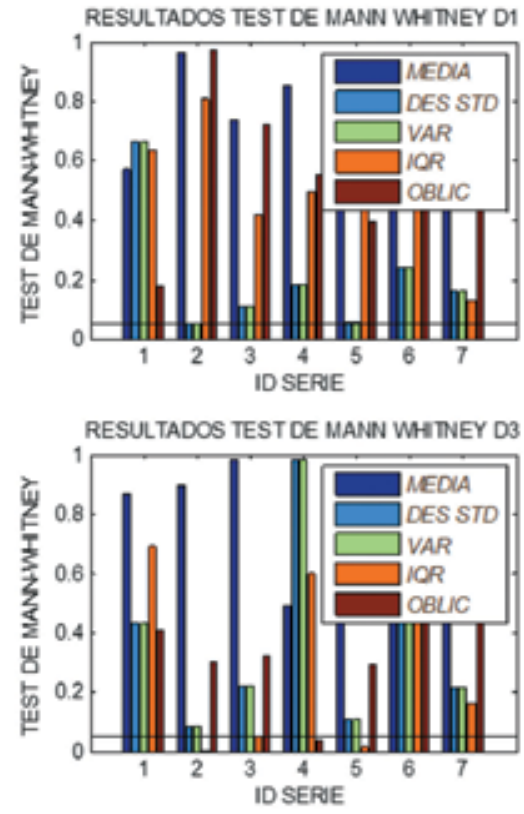

do los componentes principales que abarquen la mayor varianza de los datos, obteniendo del diagrama de Pareto que al utilizar tres componentes principales se obtiene un $99.2686 \%$ del total de la varianza.

\section{SISTEMA DE CLASIFICACIÓN}

El objetivo de un clasificador es predecir a qué clase pertenecen las muestras evaluadas, así como reducir el tiempo de ejecución ante cualquier proceso relacionado con la minería de datos.

\subsection{Clasificador K-Nearest Neighbor (K-NN)}

La idea básica sobre la que se fundamenta este algoritmo es que un nuevo caso se va a clasificar en la clase más frecuente a la que pertenecen sus $\mathrm{K}$ vecinos más cercanos. La Fig. 3 muestra de manera gráfica un ejemplo de esta técnica. Tal y como puede verse, se tienen 24 casos ya clasificados en dos posibles valores $(m=2)$. Las variables predictoras son $\mathrm{X} 1$ y X2, y se ha seleccionado $\mathrm{K}=3$ número de vecinos. De los tres casos ya clasificados que se encuentran más cercanos al nuevo caso por clasificar, representado por ', dos de ellos pertenecen a la clase $\circ$, por tanto el clasificador 3-NN predice la clase $\circ$ para el nuevo caso. 
Fig. 3. EJEMPLO DE APLICACIÓN DEL ALGORITMO K-NN BÁSICO

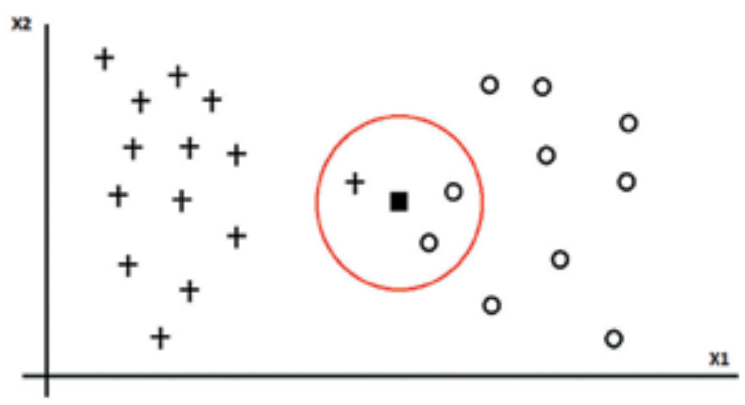

Fuente: autores

Para el diseño del clasificador K-NN se separaron las 3 variables, resultado de la aproximación lineal que entrega el PCA, en dos clases, distribuyendo un $70 \%$ de los datos para la etapa de entrenamiento y $30 \%$ para la etapa de test. Para cada grupo de datos se modificó el número de los vecinos y se analizaron cinco formas diferentes para medir la distancia entre los datos: Euclidiana, City block, Mahalanobis, Chebyshev y Minkowski. Se realizaron 150 corridas del algoritmo, variando en cada corrida los datos de entrenamiento y test, manteniendo un balance entre las clases $\mathrm{CO}$ y $\mathrm{C} 1$, obteniendo que los mejores porcentajes de clasificación se alcanzan con $\mathrm{K}=5$ y la distancia Mahalanobis. En la Tabla II se observan los porcentajes de clasificación para los cinco métodos utilizados para evaluar la distancia y tres tamaños diferentes para el número de vecinos.

TABLA II

PORCENTAJES DE CLASIFICACIÓN DEL ALGORITMO K-NN

\begin{tabular}{|l|c|c|c|}
\cline { 2 - 4 } \multicolumn{1}{c|}{} & \multicolumn{3}{c|}{ NÚMERO DE VECINOS } \\
\hline \multicolumn{1}{c|}{ DISTANCIA } & $\mathrm{K}=3$ & $\mathrm{~K}=5$ & $\mathrm{~K}=7$ \\
\hline Euclidiana & $55.66 \% \pm 0.083$ & $57.01 \% \pm 0.067$ & $53.64 \% \pm 0.152$ \\
\hline City block & $53.66 \% \pm 0.102$ & $56.76 \% \pm 0.049$ & $54.19 \% \pm 0.095$ \\
\hline Mahalanobis & $53.08 \% \pm 0.210$ & $58.10 \% \pm 0.094$ & $54.49 \% \pm 0.074$ \\
\hline Chebychev & $55.48 \% \pm 0.072$ & $57.36 \% \pm 0.081$ & $54.42 \% \pm 0.207$ \\
\hline Minkowski & $54.33 \% \pm 0.153$ & $57.25 \% \pm 0.106$ & $52.00 \% \pm 0.246$ \\
\hline
\end{tabular}

\subsection{Clasificador difuso}

La lógica difusa es una lógica alternativa a la lógica clásica que pretende introducir un grado de vaguedad en las cosas que evalúa. La lógica difusa en comparación con la lógica convencional permite trabajar con información que no es exacta para poder definir evaluaciones convencionales, contrario con la lógica tradicional que permite trabajar con información definida y precisa. En el clasificador difuso tipo sugeno se consideran los siguientes criterios:

- Funciones de pertenencia. Para cada una de las tres variables lingüísticas se definieron cuatro funciones de membresía tipo gaussiano, distribuidas inicialmente de manera simétrica y solapadas, de tal manera que cada uno de los elementos de los universos de discurso de cada variable debe pertenecer, al menos, a un conjunto difuso. Para la variable de salida se definieron funciones lineales de la forma (3), donde $a_{j}$ corresponde a la ponderación que se le da a cada variable lingüística $x_{j}$ y $b$ es una constante de umbralización.

$$
Z_{j}=\sum_{j=1}^{r} a_{j} x_{j}+b
$$

- Operadores. La evaluación del antecedente, $\mathrm{W}_{\mathrm{j}}$, se realiza con el mínimo entre los grados de pertenencia de los conjuntos relacionados en cada premisa.

- Método de inferencia. El método de inferencia empleado está dado por (4), donde el valor de $\imath$ corresponde al número de reglas establecidas para el sistema difuso, que para este problema fueron sesenta y cuatro.

$$
f=\frac{\sum_{j=1}^{l} w_{j} Z_{j}}{\sum_{j=1}^{l} w_{j}} \text { (4) }
$$

A partir de la base de datos y utilizando un algoritmo de backpropagation para minimizar el error de la aproximación, se definen los parámetros del clasificador difuso: distribución de las funciones de pertenencia en sus universos de entrada y determinación de las constantes de las funciones lineales. El algoritmo se detiene cuando se ha alcanzado el error medio cuadrático establecido o el número máximo de iteraciones. Una vez diseñado el clasificador difuso se ajustó un umbral general con el objetivo de mejorar el porcentaje de clasificación: se define un umbral y se realizan 150 corridas modificando de forma aleatoria los datos de test para cada corrida, manteniendo un balance entre las clases $\mathrm{CO}$ y $\mathrm{C} 1$. En la Fig. 4 se observa que para un valor de 0.4 se obtiene el mayor porcentaje de clasificación que es del $80.00 \% \pm 0.152$. 
Fig. 4. CURVA UMBRAL VS PORCENTAJE DE CLASIFICACIÓN

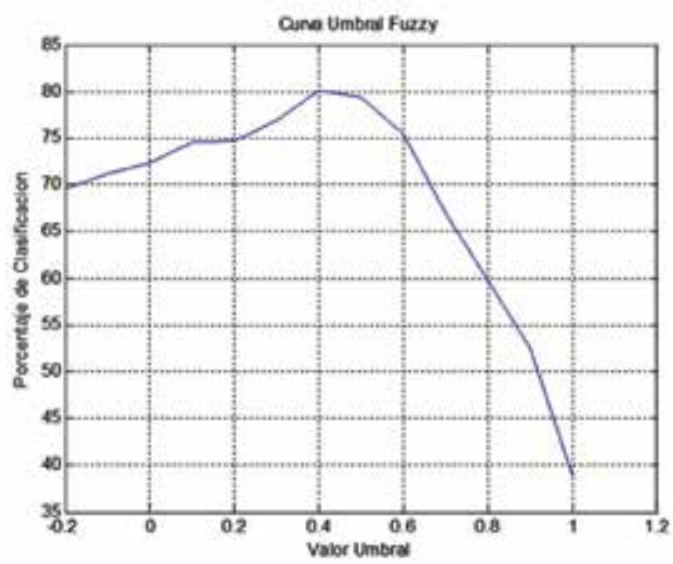

Fuente: autores.

\section{CONCLUSIONES}

El proceso de extubación de un paciente asistido mediante VM, es el último paso por seguir para recuperar la respiración espontánea. Tanto la permanencia como el retiro precoz de la ventilación mecánica provocan riesgos en el paciente, aumentando la posibilidad de contraer infecciones, atrofia de la musculatura respiratoria, pérdida de los mecanismos de defensa, adicionalmente una estancia más prolongada aumenta los costos hospitalarios para la clínica y el paciente. Por tal motivo el hecho de presentar una metodología que permita determinar si el paciente se puede desconectar del ventilador, se convierte en un sistema de apoyo para el diagnóstico médico.

La metodología presentada se fundamenta en la transformada discreta de wavelet, la cual nos permitió, determinar tres índices que caractericen el comportamiento del flujo respiratorio. La selección de la wavelet madre es un paso importante para el tratamiento a la señal, porque con ella se logra definir una mayor diferenciación entre los índices. Como sistema de clasificación se recomienda la lógica difusa, que alcanzó un porcentaje de clasificación del $80 \%$ con los datos de la base de datos WEAN DB, pero es importante al utilizar este método, utilizar previamente un algoritmo para reducir la dimensionalidad, dado que el costo computacional es alto.

\section{AGRADECIMIENTOS}

Los autores agradecen al personal de la Unidad de Cuidados Intensivos del Hospital de la
Santa Creu i Sant Pau de Barcelona y del Hospital Universitario de Getafe, Getafe, España, por su colaboración con la base de datos de la señal.

\section{REFERENCIAS}

[1] J. F. McConville, J. P. Kress, "Weaning patients from the ventilator," The new England Journal of Medicine, vol. 367, pp. 2233-9, 2012.

[2] A. Esteban, F. Frutos Vivar, A. Muriel, N. D. Ferguson, O. Peñuelas et al., "Evolution of mortality over time in patients receiving mechanical ventilation". American Journal of Respiratory and Critical Care Medicine, vol. 188, no. 2, 2013.

[3] J. F. McConville, J. P. Kress, "Weaning patients from the ventilator," New England Journal of Medicine, vol. 367, no. 23, pp. 2233-2239, 2012.

[4] G. Benchetrit, "Breathing pattern in humans: diversity and individuality," Respiration Physiology, vol. 122, pp. 123-129, 2000.

[5] P. Caminal, L. Domingo, B.F. Giraldo, M. Vallverdú, S. Benito, G. Vázquez, D. Kaplan, “Variability analysis of the respiratory volume based on nonlinear prediction methods," Medical \& Biological Engineering \& Computing, vol. 42, pp. 86-91, 2004.

[6] M. J. Tobin, M.J. Mador, S.M. Guenter, R.F. Lodato, M.A. Sackner, "Variability of resting respiratory center drive and timing in healthy subjects," J. Journal Applied Physiology, vol. 65, pp. 309-317, 1988.

[7] E. N. Bruce, "Measures of respiratory pattern variability, Bioengineering approaches to pulmonary physiology and medicine," Plenum Press, pp. 149-160, 1996.

[8] M. C. Khoo, "Determinants of ventilatory instability and variability," Respiratory physiology, vol. 122, pp. 167-182, 2000.

[9] J. A. Chaparro, B. F. Giraldo, P. Caminal, S. Benito, "Analysis of the respiratory pattern variability of patients in weaning process using autoregressive modeling techniques," Engineering in Medicine and Biology Society, EMBC, 2011.

[10] B. F. Giraldo, B. W. Gaspar, P. Caminal, S. Benito, "Analysis of roots in ARMA model for the classification of patients on weaning trials," Engineering in Medicine and Biology Society, EMBC, 2012.

[11] Y. Hao-Yung. "Using support vector machine to construct a predictive model for clinical decision-making of ventilation weaning," Neural Networks, 2008. IJCNN 2008. (IEEE World Congress on Computational Intelligence). 
[12] B. Giraldo, A. Garde, C. Arizmendi, R. Jane, S. Benito, I. Díaz, D. Ballesteros, "Support vector machine classification applied on weaning trials patients," Engineering in Medicine and Biology Society, 2006. EMBS ‘06. 28th Annual International Conference of the IEEE.

[13] B. Giraldo, C. Arizmendi, E. Romero, R. Alquezar, P. Caminal, S. Benito, D. Ballesteros, "Patients on weaning trials from mechanical ventilation classified with neural networks and feature selection," Engineering in Medicine and Biology Society, 2006. EMBS '06. 28th Annual International Conference of the IEEE.

[14] C. Arizmendi, E. Romero, R. Alquezar, P. Caminal, I. Díaz, S. Benito, B. F. Giraldo, “Data mining of patients on weaning trials from mechanical ventilation using cluster analysis and neural networks," Engineering in Medicine and Biology Society, 2009. EMBC 2009.
[15] P. Caminal, B. F. Giraldo, M. Vallverdú, S. Benito, R. Schiroeder, A. Voss, "Simbolic dynamic analysis of relations between cardiac and breathing cycles in patients on weaning trials," Annals of Biomedical Engineering, vol. 38, no. 8, August, 2010, pp. 2542-2552.

[16] L. S. Correa, E. Laciar, B. F. Giraldo, A. Torres, “Multiparameter analysis of ECG and respiratory flow signals to identify success of patients on weaning trials," 32nd Annual International Conference of the IEEE EMBS, 2010.

[17] A. Arcentales, B. F. Giraldo, P. Caminal, S. Benito, A. Voss, "Recurrence quantification analysis of heart rate variablility and respiratory flow series in patients on weaning trials," 33rd Annual International Conference of the IEEE EMBS, 2011. 